Published in final edited form as:

Semin Neurol. 2014 February ; 34(1): 7-13. doi:10.1055/s-0034-1372337.

\title{
Evolving Character of Chronic Central Nervous System HIV Infection
}

\author{
Richard W. Price, MD ${ }^{1}$, Serena S. Spudich, MD, MA², Julia Peterson, BS ${ }^{1}$, Sarah Joseph, \\ $\mathrm{PhD}^{3}$, Dietmar Fuchs, $\mathrm{PhD}^{4}$, Henrik Zetterberg, MD, $\mathrm{PhD}^{5}$, Magnus Gisslén, $\mathrm{MD}, \mathrm{PhD}^{6}$, and \\ Ronald Swanstrom, PhD $^{3}$ \\ ${ }^{1}$ Department of Neurology, University of California, San Francisco, California \\ ${ }^{2}$ Department of Neurology, Yale University, New Haven, Connecticut \\ ${ }^{3}$ UNC Center for AIDS Research and the Lineberger Comprehensive Cancer Center, University \\ of North Carolina at Chapel Hill, Chapel Hill, North Carolina \\ ${ }^{4}$ Division of Biological Chemistry, Biocenter, Innsbruck Medical University, Innsbruck, Austria \\ ${ }^{5}$ Department of Psychiatry and Neurochemistry, Institute of Neuroscience and Physiology, \\ University of Gothenburg, Gothenburg, Sweden and Institute of Neurology, Queen Square, \\ London, United Kingdom
}

${ }^{6}$ Department of Infectious Diseases, University of Gothenburg, Gothenburg, Sweden

\begin{abstract}
Human immunodeficiency virus type 1 (HIV-1) infection of the central nervous system (CNS) begins early in systemic infection and continues throughout its untreated course. Despite a common cerebrospinal fluid inflammatory response, it is usually neurologically asymptomatic for much of this course, but can evolve in some individuals to HIV-associated dementia (HAD), a severe encephalopathy with characteristic cognitive and motor dysfunction. While widespread use of combination antiretroviral therapy (ART) has led to a marked decline in both the CNS infection and its neurologic severe consequence, HAD continues to afflict individuals presenting with advanced systemic infection in the developed world and a larger number in resource-poor settings where ART is more restricted. Additionally, milder CNS injury and dysfunction have broader prevalence, including in those treated with ART. Here we review the history and evolving nomenclature of HAD, its viral pathogenesis, clinical presentation and diagnosis, and treatment.
\end{abstract}

(C) 2014 by Thieme Medical Publishers, Inc.

Address for correspondence Richard W. Price, MD, Department of Neurology, University of California San Francisco, Bldg 1 Room 101, San Francisco General Hospital, Box 08701001 Potrero Avenue, San Francisco, CA 94110 (e-mail: rwprice@sfgh.ucsf.edu).

Conflict of Interest

RWP is a consultant to Merck and Co and has received an honorarium and travel support from Abbott Laboratories for meeting presentation. MG has received research grants from Abbott, Baxter, Bristol-Myers Squibb, Gilead Sciences, GlaxoSmithKline, Merck, Pfizer, Roche, and Tibotec. He has received honoraria as speaker and/or advisor from Abbott/Abbvie, Bioinvent, BoehringerIngelheim, Bristol-Myers Squibb, Gilead Sciences, GlaxoSmithKline, Janssen-Cilag, Merck, Pfizer, Roche, and Tibotec/Janssen. SS has received an honorarium and travel support from AbbVie, Inc. 


\section{Keywords}

HIV; dementia; treatment; brain; inflammation; cerebrospinal fluid; biomarkers

Infection of the central nervous system (CNS) is a universal facet of systemic human immunodeficiency virus-1 (HIV) infection. It begins shortly after exposure during initial systemic viremia ${ }^{1-3}$ and continues through the subsequent course of chronic untreated systemic infection and disease. ${ }^{4,5}$ Importantly, the character and neurologic consequences of this infection vary considerably among individuals and evolve over a protracted chronic course, changing from seemingly innocent meningitis inmost patients to severe encephalitis in a substantial minority late in systemic disease. ${ }^{6}$ In this review, we outline the general characteristics of this infection, drawing mainly on findings in cerebrospinal fluid (CSF) that have provided unique insight into the dynamics and evolving pathogenesis of this chronic process. $^{7}$

CNS infection is an offshoot of systemic infection that determines or influences events in the CNS in several ways. Systemic viremia provides initial and likely continuous seeding of blood-borne virus into the CNS, while in the background its effects on lymphocyte populations orchestrate the changes in host immune function, inducing both immunosuppression and immune activation that in turn further modulate not only systemic infection and disease evolution, ${ }^{8}$ but also the course of CNS infection and injury. ${ }^{9}$ Although HIV infects mainly CD4+ T lymphocytes, importantly it can also infect cells of the monocyte-macrophage-microglia lineage; as infection evolves, its main CNS targets may change from lymphocytes to macrophages and related cells. ${ }^{10}$

In this review, we consider different stages in the evolution of untreated CNS infection: early, or primary HIV infection (PHI) spanning the first year after exposure and initial viremia; evolving chronic neuro-asymptomatic infection (NA) that changes as the immune system is progressively altered and blood CD4+ T cells fall with few or relatively mild neurologic symptoms and signs; and overt HIV-associated dementia (HAD) presenting with subacute onset and progression of substantial cognitive-motor dysfunction. ${ }^{11}$ This temporal separation provides a convenient framework for examining the evolving pathobiology of untreated CNS infection (treatment of infection is considered elsewhere in this issue). Before discussing this evolution, we will first briefly consider the main interacting-disease components and their biomarker indicators in CSF and blood.

\section{Evolving CNS Infection and CSF Biomarkers}

Fig. 1 presents a simplified model for considering CNS HIV infection and disease evolution in relation to their systemic counterparts. ${ }^{7}$ The top of the figure is a diagram of the systemic interaction of HIV and the host immune system: infection evolves over time with selection and expansion of viral populations in concert with changes in the immune system that exhibits both progressive deficiency and broad activation. ${ }^{12}$ Immunodeficiency leads to an array of systemic diseases, most notably opportunistic infections that define acquired immunodeficiency syndrome (AIDS), while immune activation is also critical to systemic progression and likely contributes importantly to organ injuries including a variety of non- 
AIDS-related diseases. ${ }^{8,13-15}$ These systemic interactions are now routinely monitored by blood biomarkers. Thus, plasma HIV RNA levels are used to predict the rate of disease progression and response to antiviral therapy, while the blood CD4+ $\mathrm{T}$ cell count provides an index of the cumulative damage to the immune system and the vulnerability to opportunistic diseases. ${ }^{16}$ Soluble and lymphocyte phenotype markers that assess systemic immune activation also predict disease progression and risk. ${ }^{17-19}$ These viral and immune biomarkers have provided invaluable insight into the biology and natural history of HIV infection, and their incorporation into standard practice has had a profound effect on the clinical management and therapeutics of HIV infection.

The downward vertical arrows in Fig. 1 that cross the dotted line (representing the bloodbrain and blood-CSF barriers) emphasize the fact that both CNS HIV infection and immune-inflammatory responses originate from their systemic counterparts with selective transfer from the blood into the CNS of infection (including both infected cells and uninfected target CD4+ T cells) and both virus-specific immune and broader inflammatory reactions. ${ }^{20-22}$ Subsequently, each of these CNS-disease components can diverge from their systemic origins, producing a distinct infected and reactive milieu within the CNS, including the leptomeninges, perivascular compartment, and brain parenchyma. Additionally, both the infection itself and immune-inflammatory reactions can impact nervous system integrity and function, ${ }^{9}$ thus forming a pathogenic triangle with two main interacting agonists and a target as diagramed below the dotted line in Fig. 1.

In the following sections we will examine changes in these three components through the course of evolving infection, illustrated with some of our own data from a cross-sectional study of CSF biomarkers (Fig. 2). ${ }^{7}$ Here we focus on a few biomarkers: blood CD4+ T cell count and plasma HIV RNA as indices of systemic infection, CSF HIV RNA as a measure of the magnitude of CNS infection, CSF white blood cell (WBC) count (consisting mainly of T lymphocytes ${ }^{22}$ ) and neopterin (a pteridine metabolite produced within the CNS primarily by cells of the monocyte-macrophage-microglial lineage, but also by astrocyte $^{23,24}$ ) as measures of inflammation; and CSF neurofilament light-chain protein (NFL) as a sensitive indicator of active brain injury. ${ }^{25}$ We will examine how these components change over the course of infection. After this, we will consider how these changes might relate to the evolving genetic and phenotypic character of CNS HIV infection. Finally, we will also briefly consider how these biomarkers might be used in clinical practice.

\section{The CNS in Primary Infection}

HIV seeds the CNS very soon after initial inoculation during primary systemic viremia at a time when systemic infection is marked by an early reduction of blood CD4+ T cells (Fig. 2A) and elevation of CD8+ cells (not shown). Simple comparisons of PHI with HIV groups shows alteration of both T-cell subsets in PHI by Mann-Whitney ( $p=0.0028$ for CD4+ and $<0.0001$ for CD8+ cells ${ }^{\mathrm{a}}$ ). This initial phase is variable among patients and may establish

\footnotetext{
aAll statistical comparisons described here use nonparametric methods and were performed in Prism 6.0 (Graph Pad Software, Inc., San Diego, CA).
} 
the basic character and trajectory of subsequent infection. It is also sufficiently different from later chronic infection, to be considered separately. Despite levels of plasma HIV RNA comparable to those found later (Fig. 2B), the magnitude of CSF HIV RNA concentration is generally relatively low compared with that of plasma in this early phase (Fig. 2C). Thus, in PHI the CSF HIV RNA is generally closer to 100-fold lower than plasma compared with the 10-fold lower level characteristic of later chronic infection (except in those with blood CD4+ counts below50 cells/ $\mu \mathrm{L}$ as discussed below) (ratios between CSF and plasma HIV concentrations in PHI differed from the three NA groups with higher CD4+ T cell counts using Kruskal-Wallis test and Dunn's post comparisons, $p<0.05$ to 0.001 , but not from NA CD4+ T cells $<50$ ). The reason for this relatively lower CSF HIV RNA level is not clear, but presumably reflects more restricted entry of infected CD4+ T cells or subsequent viral amplification within the CSF space (leptomeninges) in this early stage. Though immune activation and inflammation appear to be generally important in augmenting CNS infection, the elevated CSF WBC counts and neopterin levels in these patients (Fig. 2D and E) are similar to those of chronic infection with CD4 $\mathrm{T}$ cells above $350 \mathrm{cells} / \mu \mathrm{L}$, and thus these simple measures do not provide a ready explanation for the relatively lower CSF viral levels in PHI. Perhaps early antiviral immune responses can partially suppress this infection within the CNS.

Of note, more than $25 \%$ of PHI patients exhibit mild elevations of CSF NFL (Fig. 2F), indicating an initial phase of CNS injury ( $p=0.0076$ by Mann-Whitney comparing PHI to HIV-groups). ${ }^{26}$ Presumably, this is caused by mechanisms similar to those responsible for injury later in the course, including viral and inflammatory processes. However, it is not clear why this occurs during this early period, but is less prevalent subsequently in patients chronically infected with similar blood CD4+ T cell counts, CSF viral loads and inflammatory responses. Whatever mechanismis involved, elevated CSF NFL concentrations demonstrate that there is a clear impact of initial infection on the brain that potentially might have lasting consequences despite its resolution.

\section{Evolving Chronic Infection}

The relations among CSF HIV, inflammation and neural injury change as systemic disease progresses and blood CD4+ T cells fall. As shown in the results of the four NA groups in Fig. 2, plasma virus levels rose as CD4+T cells fell below350 cells/ $\mu \mathrm{L}$, returning to the high levels noted in the PHI group. However, CSF HIV RNA followed a different pattern-also rising as CD4 $\mathrm{T}$ cells fell below 350, but then falling when CD4+ T cells were reduced to below 50 cells $/ \mu \mathrm{L}$, providing a second setting characterized by a CSF: plasma ratio near 1:100. However, in contrast to the PHI group, the CSF WBC count was also markedly attenuated in these patients (differing from all the other infected groups, $p<0.01$ to 0.0001 by Kruskal-Wallis and Dunn's correction for multiple comparisons), suggesting that reduced CSF T-cell traffic might be important in limiting local infection, either by effects on the influx of infected cells or the main cell targets capable of amplifying locally replicating virus in the meninges. Despite the lack of lymphocytic inflammation, CSF neopterin levels were similar to those of patients with blood CD4+ cells $>50$ cells/ $\mu \mathrm{L}$, indicating divergence among different components of inflammation in this setting. Indeed, this dissociation of WBCs and neopterin suggests that macrophage inflammation in the CNS may have 
continued while lymphocytic inflammation was waning with the severe decrease in CD4+ T cells in these individuals.

Accompanying the lowered CSF HIV RNA and WBC counts in the group with blood CD4 + $\mathrm{T}$ cells $<50$ cells $/ \mu \mathrm{L}$ was a substantial incidence of abnormal CSF NFL concentrationsnearly $75 \%$ of subjects in this group had elevations of this neuronal injury biomarker. An increase was also noted in $>25 \%$ of subjects with blood CD4+ T cells between 50 and 200 cells $/ \mu \mathrm{L}$, indicating substantial development of subclinical CNS injury in patients with blood CD4+cells that define an AIDS diagnosis despite the absence of conspicuous neurologic symptoms or signs. Vulnerability to serious opportunistic infections is thus paralleled by sometimes cryptic neuropathogenic CNS HIV infection. In this cross-sectional study, neither CSF HIV RNA, WBC counts nor neopterin clearly predicted this injury, though neopterin has correlated more closely in larger cohorts. ${ }^{23}$

\section{HIV-Associated Dementia}

Although formal diagnostic criteria for HAD in the research setting now include poor performance on extensive neuropsychological testing ${ }^{27}$ the clinical presentation of these cases generally conforms to the early descriptions of AIDS dementia complex (ADC) with subacute evolution of distinct and disabling cognitive-motor dysfunction. ${ }^{11}$ The profile of CSF biomarkers in our study suggest that many of these patients show pathogenetic distinctions or at least depart from those with less symptomatic neurologic dysfunction with respect to certain CSF features, including higher HIV RNA, WBC counts, neopterin, and NFL.

Like subclinical injury, overt HAD predominantly develops in patients with $<200$ blood CD4+ T cells $/ \mu \mathrm{L} .5,6$ In the illustrated study, both plasma and CSF HIV RNA levels were high in the HAD group, but CSF HIV levels were not above those of many NA patients, including those with higher blood CD4+ counts. Likewise, CSF pleocytosis was common, contrasting with the NA group with $<50$ blood CD4+ T cells/ $\mu \mathrm{L}$ ( $p<0.01$ by Dunn's multiple comparison test after Kruskal-Wallis test), but not with many of the NA subjects with higher CD4 counts. CSF neopterin was notably elevated and NFL was consistently very high, distinguishing this group. Thus, in this sample of HAD, robust inflammation was often present and included both neopterin (macrophage) and WBC (lymphocyte) markers. This inflammatory profile may be important in determining the particular severity and clinical phenotype of these patients compared with those with less-apparent neural injury.

\section{Evolving Genetic and Phenotypic Character of CNS Infection}

The discussion above centered on the quantitative changes in CSF biomarkers, including HIV RNA, without providing a clear explanation of what determines onset of neurologic injury. Additional, likely critical factors in CNS disease are (1) the development of independent local viral replication within the CNS, termed compartmentalized infection; and (2) change in the phenotypic character of these compartmentalized viruses allowing them to more readily infect macrophages and related cells that express low levels of the CD4 receptor on their surface, macrophage- (M-) tropism(Fig. 3). ${ }^{28-30}$ These infected 
macrophages may be the potent initiators of neuropathogenic pathways that are further amplified by uninfected macrophages and other cells. ${ }^{31}$

Early in infection, most notably during primary infection, CSF viral isolates are virtually identical to those found simultaneously in blood plasma. This is noncompartmentalized infection and presumably reflects the origin of these CSF viral populations from bloodborne CD4+ T lymphocytes characterized by recent entry and limited life span. Virus isolates during this period are T-tropic and use the CCR5 coreceptor (hence, termed R5 viruses). Subsequently, after at least several months and more commonly during later evolving chronic infection, CSF viral populations examined by direct cloning and phylogenetic analysis can show evidence of bursts of local replication from a common ancestor, so-called clonal expansion, which is one type of compartmentalized infection. These appear to be relatively transient events, but confirm the capacity of the CNS to support independent replication and viral amplification. These viral populations remain Tcell tropic and R5, indicating that these expansions are supported within CD4+ T cells that have entered the CNS, residing largely in the meninges but perhaps also in the perivascular spaces. At times these amplifications will further evolve to somewhat more complex diversification, though still as T-cell tropic populations.

However, in some patients, most characteristically those presenting with HAD, further viral evolution will produce more complex populations of M-tropic viruses that can replicate in cells expressing low levels of the CD4 viral receptor. These continue to use the CCR5 coreceptor, associate with a macrophage-related inflammatory responses and appear to be more neuropathogenic through inflammatory signals that alter brain function and structural integrity by indirect mechanisms. ${ }^{6,9}$ With immunosuppression, blood viruses remain Ttropic, but in some patients an additional viral lineage will appear that has switched its coreceptor preference to CXCR4 and this is associated with more rapid progression of immunosuppression, although cause and effect are still unclear. ${ }^{32}$ These T-tropic X4 viruses (or commonly dual-tropic viruses with capacity to interact with both CCR5 and CXCR4 coreceptors) may also appear in the CSF, presumably carried within infected CD4+T cells, but have not been associated with HAD, though data on this issue are limited. ${ }^{33}$

How does this viral evolution reconcile with the evolving patterns of CSF HIV RNA concentrations, inflammation and NFL elevations? Answering this takes us further into the realm of speculation, and invites future study to address this critical question. In some patients with HAD the role of M-tropic viruses seems assured: not only do the predominant viral populations found in the CSF exhibit the M-tropic phenotype, but they also shows low decay in the face of antiviral therapy, implying production from a long-lived cell such as a macrophage, in contrast to T-cell-tropic viruses, which decay at rapid a rate (being produced from short-lived T cells) similar to those of plasma, just as is seen in nondemented patients. $5,29,34,35$ The relatively low CSF HIV RNA content and absence of pleocytosis despite rising CSF NFL in the NA groups with low blood CD4+ T cells likely indicates inflammation driven by an expanding infection of macrophages and related cells without an inflowing CD4+ T-cell population to contribute to the local viral load or provide target cells for amplification of the either T- or M-tropic viruses. However, some patients with HAD exhibit rapid decay and T-tropic viruses in CSF. ${ }^{29}$ There are at least two plausible 
explanations: (1) T-tropic CNS HIV infection can also cause neurologic injury, and thus, Mtropism is not a strict requirement; or (2)M-tropic viruses may still be involved in neural injury, but may evoke a T-cell inflammatory response with pleocytosis in that influx of $\mathrm{T}$ cells brings in T-tropic viruses that obscure the underlying neuropathogenic infection. These are not mutually exclusive mechanisms and could explain the greater range of pleocytosis and high CSF HIV RNA in our HAD patients that contrast with the NA with CD4 cells below 50. In our previous small study, there was a trend toward higher CD4+ T cell counts and pleocytosis in the HAD subjects with T-cell-tropic virus compared with those with Mtropic virus in the CSF. ${ }^{29}$

\section{Clinical Diagnosis and Management}

At present, diagnosis of CNS disease related to HIV infection involves a two-pronged approach: (1) to demonstrate the presence and severity of neurologic dysfunction, and (2) to implicate HIV as the likely cause. The first uses the neurologic history and examination, but to conform to formal research criteria also entails rather detailed neuropsychological testing depending on the severity and setting. ${ }^{27}$ The second often focuses on ruling out other causes by history (e.g., active drug use, past head trauma or other neurological insults, etc.) or by laboratory testing including neuroimaging and CSF analysis primarily focused on detecting alternative diagnoses that account for the clinical phenotype. ${ }^{36}$ It is reasonable to ask if the biomarker patterns we have observed can help in diagnosis and management of patients.

Currently, CSF neopterin and NFL are not generally available for real-time clinical use, though we suggest that they should be. Other, more readily available, inflammatory markers with similar characteristics might be substituted for the former, for example, CSF $\beta$-2microglobulin $\left(\beta_{2} \mathrm{M}\right) .{ }^{37}$ However, like neopterin, $\beta_{2} \mathrm{M}$ has limited diagnostic specificity in distinguishing $\mathrm{HAD}$ from opportunistic infections. It might be helpful in distinguishing active from static brain injury from past causes, but because of elevations related to meningeal inflammation, this use is probably largely applicable to treated patients rather than those off treatment. ${ }^{38}$ On the other hand, CSF NFL we feel may be more useful in this regard. It would more sensitively detect the presence or absence of CNS injury and thus active from static CNS injury, though again without distinguishing cause. ${ }^{39}$ Nonetheless, one can readily see where such a test could be used in HIV infection.

On the other hand, given the state of therapy and evolving guidelines, the direct need for these distinctions may be eclipsed by the broader call for earlier antiretroviral treatment for all viremic patients, at least in theory. As discussed elsewhere in this volume, this may only be important if CNS disease is to be treated with different drug regimens than currently recommended therapies that have been based on systemic treatment characteristics. ${ }^{40,41}$ Still, more than $50 \%$ of HIV-patients in many centers in the United States and Europe, and even higher proportions in resource-poor areas of the world, are currently diagnosed as socalled late presenters with low CD4 cell counts $(<350 / \mathrm{L})^{42,43}$ and accordingly with an increased risk of neurocognitive disease. In these patients, monitoring neurologic status may be helpful. In those diagnosed earlier, their utility might be largely confined to (1) monitoring the group of patients who still choose not to be on therapy for evidence of advancing neuronal damage that might persuade them to begin treatment, (2) evaluating and 
monitoring individuals with neuroasymptomatic CNS escape, ${ }^{44}$ and (3) assessing ongoing low-level replication in the CNS of patients on therapy with chronic neurologic symptoms and signs. ${ }^{45,46}$

\section{Conclusions}

Chronic CNS exposure and likely infection by HIV is a nearly ubiquitous facet of systemic infection that may evolve in parallel with systemic infection, but may also pursue an independent course with respect to CNS injury and neurologic manifestations. Recent studies using CSF biomarkers and molecular analysis of viral evolution and phenotypes has led to clearer understanding of this special aspect of HIV infection and its consequences. The details of this evolution are not only biologically interesting, but have implications for the understanding neuropathogenesis and for therapeutic mitigation of disease.

\section{Acknowledgments}

Funding

Our work related to this review was supported by National Institutes of Health grants R01 MH62701, R21MH096619, R01 MH081772, P01 DA026134, P01 MH094177, UL1 TR000004 (the UCSF CTSI), Sahlgrenska Academy at the University of Gothenburg (ALFGBG-11067), the Swedish Research Council (20077092).

\section{References}

1. Davis LE, Hjelle BL, Miller VE, et al. Early viral brain invasion in iatrogenic human immunodeficiency virus infection. Neurology. 1992; 42(9):1736-1739. [PubMed: 1513462]

2. Spudich S, Gisslen M, Hagberg L, et al. Central nervous system immune activation characterizes primary human immunodeficiency virus 1 infection even in participants with minimal cerebrospinal fluid viral burden. J Infect Dis. 2011; 204(5):753-760. [PubMed: 21844301]

3. Valcour V, Chalermchai T, Sailasuta N, et al. RV254/SEARCH 010 Study Group. Central nervous system viral invasion and inflammation during acute HIV infection. J Infect Dis. 2012; 206(2):275282. [PubMed: 22551810]

4. Ellis RJ, Hsia K, Spector SA, et al. HIV Neurobehavioral Research Center Group. Cerebrospinal fluid human immunodeficiency virus type 1 RNA levels are elevated in neurocognitively impaired individuals with acquired immunodeficiency syndrome. Ann Neurol. 1997; 42(5):679-688. [PubMed: 9392566]

5. Spudich SS, Nilsson AC, Lollo ND, et al. Cerebrospinal fluid HIV infection and pleocytosis: relation to systemic infection and antiretroviral treatment. BMC Infect Dis. 2005; 5:98. [PubMed: 16266436]

6. Price RW, Brew B, Sidtis J, Rosenblum M, Scheck AC, Cleary P. The brain in AIDS: central nervous system HIV-1 infection and AIDS dementia complex. Science. 1988; 239(4840):586-592. [PubMed: 3277272]

7. Price RW, Peterson J, Fuchs D, et al. Approach to cerebrospinal fluid (CSF) biomarker discovery and evaluation in HIV infection. J Neuroimmune Pharmacol. 2013; 8(5):1147-1158. [PubMed: 23943280]

8. Deeks SG, Tracy R, Douek DC. Systemic effects of inflammation on health during chronic HIV infection. Immunity. 2013; 39(4):633-645. [PubMed: 24138880]

9. Spudich S, González-Scarano F. HIV-1-related central nervous system disease: current issues in pathogenesis, diagnosis, and treatment. Cold Spring Harb Perspect Med. 2012; 2(6):a007120. [PubMed: 22675662]

10. Burdo TH, Lackner A, Williams KC. Monocyte/macrophages and their role in HIV neuropathogenesis. Immunol Rev. 2013; 254(1):102-113. [PubMed: 23772617] 
11. Navia BA, Jordan BD, Price RW. The AIDS dementia complex: I. Clinical features. Ann Neurol. 1986; 19(6):517-524. [PubMed: 3729308]

12. Hunt PW. HIV and inflammation: mechanisms and consequences. Curr HIV/AIDS Rep. 2012; 9(2):139-147. [PubMed: 22528766]

13. El-Sadr WM, Lundgren J, Neaton JD, et al. Strategies for Management of Antiretroviral Therapy (SMART) Study Group. CD4+ count-guided interruption of antiretroviral treatment. N Engl J Med. 2006; 355(22):2283-2296. [PubMed: 17135583]

14. El-Sadr WM, Grund B, Neuhaus J, et al. SMART Study Group. Risk for opportunistic disease and death after reinitiating continuous antiretroviral therapy in patients with HIV previously receiving episodic therapy: a randomized trial. Ann Intern Med. 2008; 149(5):289-299. [PubMed: 18765698]

15. Phillips AN, Neaton J, Lundgren JD. The role of HIV in serious diseases other than AIDS. AIDS. 2008; 22(18):2409-2418. [PubMed: 19005264]

16. Mellors JW, Muñoz A, Giorgi JV, et al. Plasma viral load and CD4+ lymphocytes as prognostic markers of HIV-1 infection. Ann Intern Med. 1997; 126(12):946-954. [PubMed: 9182471]

17. Lyles RH, Tang AM, Smit E, et al. Virologic, immunologic, and immune activation markers as predictors of HIV-associated weight loss prior to AIDS. Multicenter AIDS Cohort Study. J Acquir Immune Defic Syndr. 1999; 22(4):386-394. [PubMed: 10634201]

18. Giorgi JV, Lyles RH, Matud JL, et al. Multicenter AIDS Cohort Study. Predictive value of immunologic and virologic markers after long or short duration of HIV-1 infection. J Acquir Immune Defic Syndr. 2002; 29(4):346-355. [PubMed: 11917238]

19. Neaton JD, Neuhaus J, Emery S. Soluble biomarkers and morbidity and mortality among people infected with HIV: summary of published reports from 1997 to 2010. Curr Opin HIV AIDS. 2010; 5(6):480-490. [PubMed: 20978391]

20. Shacklett BL, Cox CA, Wilkens DT, et al. Increased adhesion molecule and chemokine receptor expression on CD8+ T cells trafficking to cerebrospinal fluid in HIV-1 infection. J Infect Dis. 2004; 189(12):2202-2212. [PubMed: 15181567]

21. Sadagopal S, Lorey SL, Barnett L, et al. Enhancement of human immunodeficiency virus (HIV)specific CD8+ T cells in cerebrospinal fluid compared to those in blood among antiretroviral therapy-naive HIV-positive subjects. J Virol. 2008; 82(21):10418-10428. [PubMed: 18715919]

22. Ho EL, Ronquillo R, Altmeppen H, Spudich SS, Price RW, Sinclair E. Cellular composition of cerebrospinal fluid in HIV-1 infected and uninfected subjects. PLoS ONE. 2013; 8(6):e66188. [PubMed: 23822975]

23. Hagberg L, Cinque P, Gisslen M, et al. Cerebrospinal fluid neopterin: an informative biomarker of central nervous system immune activation in HIV-1 infection. AIDS Res Ther. 2010; 7:15. [PubMed: 20525234]

24. Yilmaz A, Yiannoutsos CT, Fuchs D, et al. Cerebrospinal fluid neopterin decay characteristics after initiation of antiretroviral therapy. J Neuroinflammation. 2013; 10:62. [PubMed: 23664008]

25. Abdulle S, Mellgren A, Brew BJ, et al. CSF neurofilament protein (NFL) - a marker of active HIV-related neurodegeneration. J Neurol. 2007; 254(8):1026-1032. [PubMed: 17420923]

26. Peluso MJ, Meyerhoff DJ, Price RW, et al. Cerebrospinal fluid and neuroimaging biomarker abnormalities suggest early neurological injury in a subset of individuals during primary HIV infection. J Infect Dis. 2013; 207(11):1703-1712. [PubMed: 23460748]

27. Antinori A, Arendt G, Becker JT, et al. Updated research nosology for HIV-associated neurocognitive disorders. Neurology. 2007; 69(18):1789-1799. [PubMed: 17914061]

28. Schnell G, Spudich S, Harrington P, Price RW, Swanstrom R. Compartmentalized human immunodeficiency virus type 1 originates from long-lived cells in some subjects with HIV-1associated dementia. PLoS Pathog. 2009; 5(4):e1000395. [PubMed: 19390619]

29. Schnell G, Joseph S, Spudich S, Price RW, Swanstrom R. HIV-1 replication in the central nervous system occurs in two distinct cell types. PLoS Pathog. 2011; 7(10):e1002286. [PubMed: 22007152]

30. Arrildt KT, Joseph SB, Swanstrom R. The HIV-1 env protein: a coat of many colors. Curr HIV/ AIDS Rep. 2012; 9(1):52-63. [PubMed: 22237899] 
31. González-Scarano F, Martín-García J. The neuropathogenesis of AIDS. Nat Rev Immunol. 2005; 5(1):69-81. [PubMed: 15630430]

32. Weiser B, Philpott S, Klimkait T, et al. Swiss HIV Cohort Study. HIV-1 coreceptor usage and CXCR4-specific viral load predict clinical disease progression during combination antiretroviral therapy. AIDS. 2008; 22(4):469-479. [PubMed: 18301059]

33. Spudich SS, Huang W, Nilsson AC, et al. HIV-1 chemokine coreceptor utilization in paired cerebrospinal fluid and plasma samples: a survey of subjects with viremia. J Infect Dis. 2005; 191(6):890-898. [PubMed: 15717264]

34. Staprans S, Marlowe N, Glidden D, et al. Time course of cerebrospinal fluid responses to antiretroviral therapy: evidence for variable compartmentalization of infection. AIDS. 1999; 13(9): 1051-1061. [PubMed: 10397535]

35. Ellis RJ, Gamst AC, Capparelli E, et al. Cerebrospinal fluid HIV RNA originates from both local CNS and systemic sources. Neurology. 2000; 54(4):927-936. [PubMed: 10690988]

36. Spudich, S.; Price, RW. Neurologic disease. In: Dolin, R.; Masur, H.; Saag, MS., editors. AIDS therapy. 3rd ed. Philadelphia, PA: Churchill Livingston Elsevier; 2008. p. 1075-1101.

37. Brew BJ, Bhalla RB, Paul M, et al. Cerebrospinal fluid beta 2-microglobulin in patients with AIDS dementia complex: an expanded series including response to zidovudine treatment. AIDS. 1992; 6(5):461-465. [PubMed: 1616651]

38. Gisslén M, Rosengren L, Hagberg L, Deeks SG, Price RW. Cerebrospinal fluid signs of neuronal damage after antiretroviral treatment interruption in HIV-1 infection. AIDS Res Ther. 2005; 2:6. [PubMed: 16109178]

39. Gisslen M, Hagberg L, Rosengren L, et al. Defining and evaluating HIV-related neurodegenerative disease and its treatment targets: a combinatorial approach to use of cerebrospinal fluid molecular biomarkers. J Neuroimmune Pharmacol. 2007; 2(1):112-119. [PubMed: 18040834]

40. Letendre S, Marquie-Beck J, Capparelli E, et al. CHARTER Group. Validation of the CNS penetration-effectiveness rank for quantifying antiretroviral penetration into the central nervous system. Arch Neurol. 2008; 65(1):65-70. [PubMed: 18195140]

41. Yilmaz A, Price RW, Gisslén M. Antiretroviral drug treatment of CNS HIV-1 infection. J Antimicrob Chemother. 2012; 67(2):299-311. [PubMed: 22160207]

42. Dennis AM, Napravnik S, Seña AC, Eron JJ. Late entry to HIV care among Latinos compared with non-Latinos in a southeastern US cohort. Clin Infect Dis. 2011; 53(5):480-487. [PubMed: 21844031]

43. Likatavicius G, Van de Laar M. HIV and AIDS in the European Union, 2011. Euro Surveill. 2012; 17(48):20329. [PubMed: 23218388]

44. Edén A, Fuchs D, Hagberg L, et al. HIV-1 viral escape in cerebrospinal fluid of subjects on suppressive antiretroviral treatment. J Infect Dis. 2010; 202(12):1819-1825. [PubMed: 21050119]

45. Canestri A, Lescure FX, Jaureguiberry S, et al. Discordance between cerebral spinal fluid and plasma HIV replication in patients with neurological symptoms who are receiving suppressive antiretroviral therapy. Clin Infect Dis. 2010; 50(5):773-778. [PubMed: 20100092]

46. Peluso MJ, Ferretti F, Peterson J, et al. Cerebrospinal fluid HIV escape associated with progressive neurologic dysfunction in patients on antiretroviral therapy with well controlled plasma viral load. AIDS. 2012; 26(14):1765-1774. [PubMed: 22614889]

47. Price RW, Epstein LG, Becker JT, et al. Biomarkers of HIV-1 CNS infection and injury. Neurology. 2007; 69(18):1781-1788. [PubMed: 17967994] 


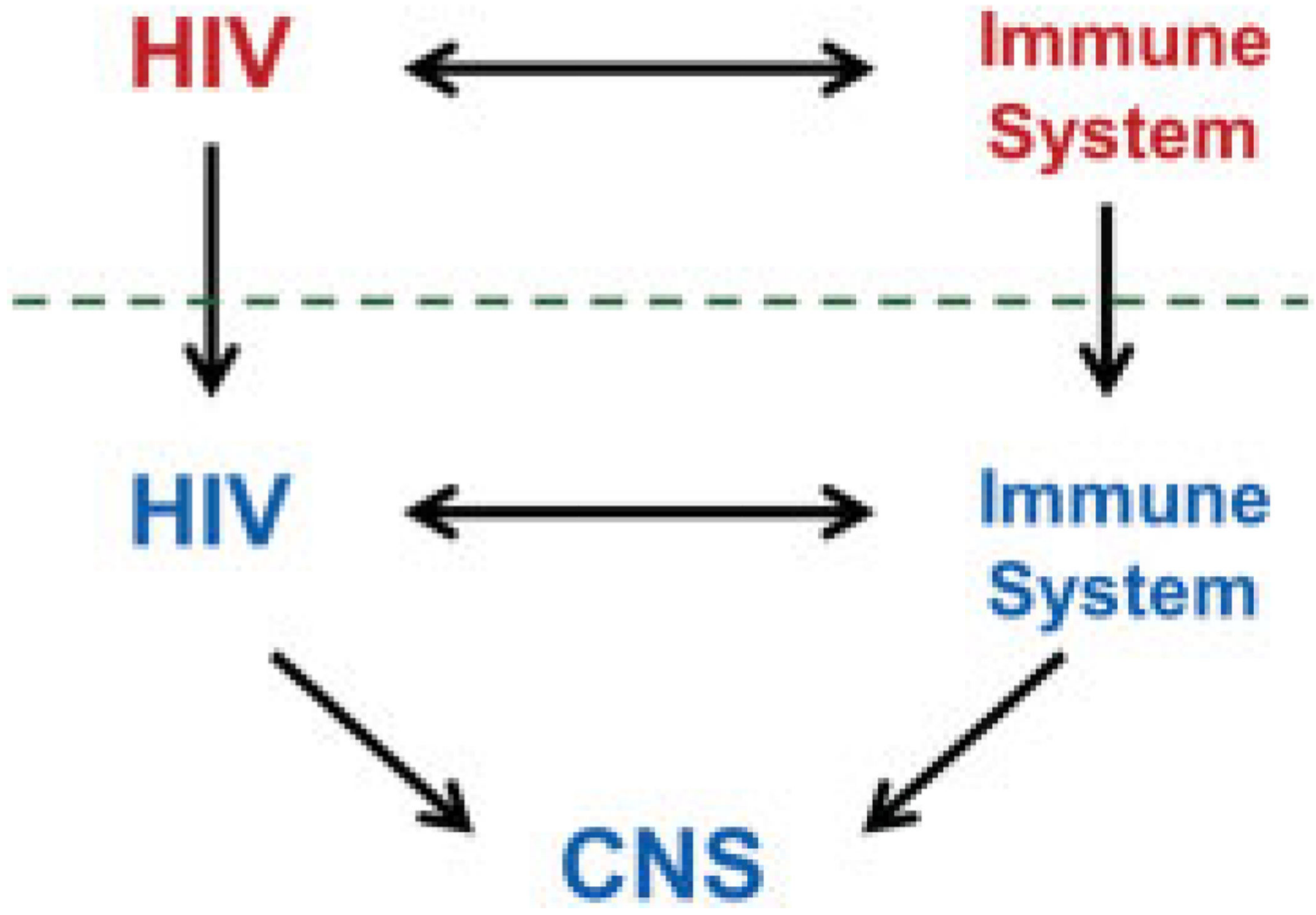

Fig. 1.

Schematic diagram of the salient systemic and central nervous system (CNS) disease components of human immunodeficiency virus (HIV) infection. CNS HIV infection and immune responses originate as extensions across the blood-brain and blood-cerebrospinal fluid barriers (horizontal dashed line) of their systemic counterparts, though with variable selection and local evolution depending on the stages of disease, and likely individual host factors. Within the nervous system, both the virus and immune responses may alter the integrity and function of the CNS in the depicted triangle of pathogenetic components. 

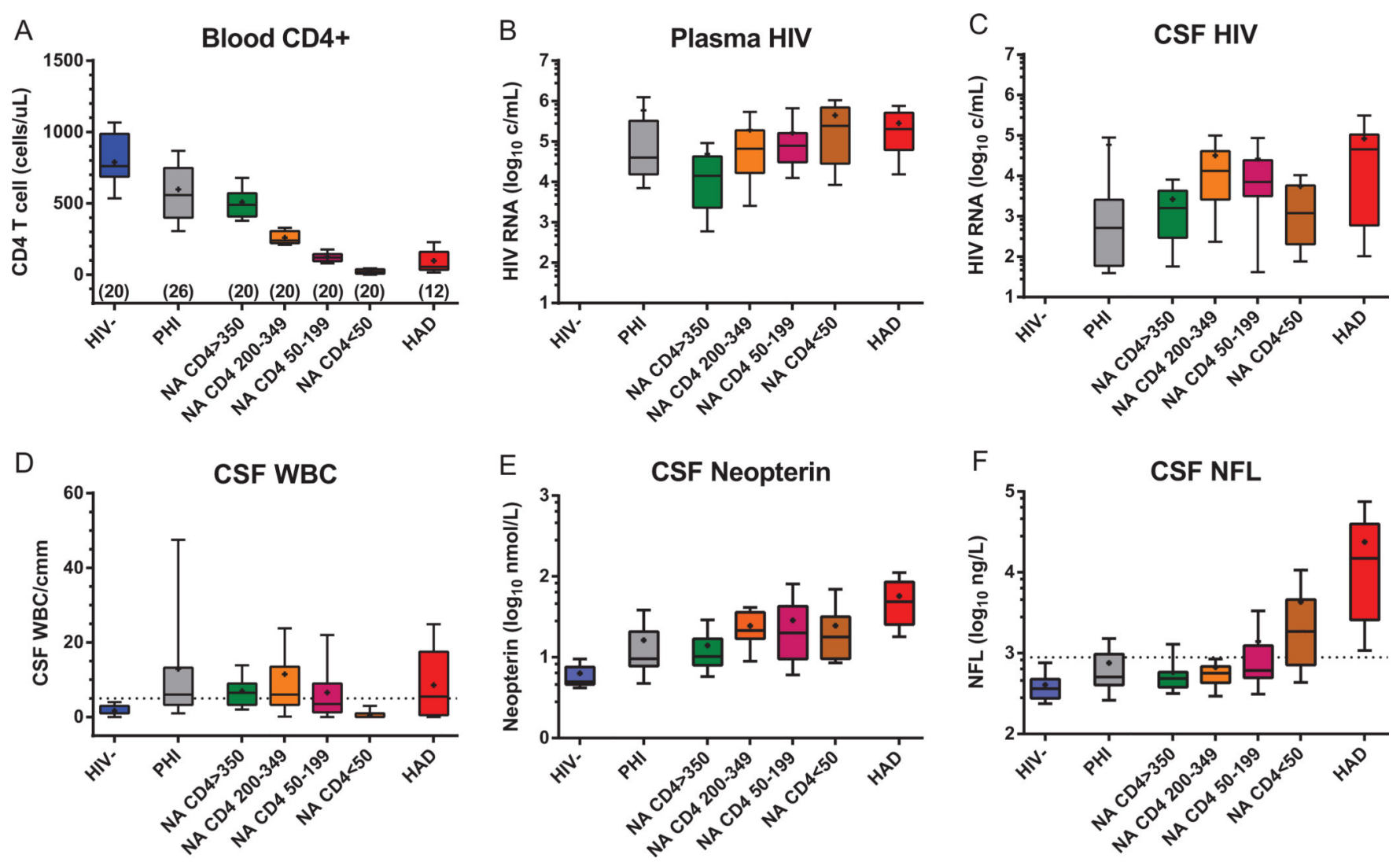

Fig. 2.

Changes in blood and cerebrospinal fluid (CSF) biomarkers with disease progression. The panels show the changes in key biomarkers across seven subject groups as described in the text. The blood CD4 counts (A) define the division of the four neuroasymptomatic (NA, includes neurologically normal and those without overt or clinically recognized neurologic complaints before recruitment into the cohort) and shows the early reduction during PHI and the low counts in the HIV-associated dementia (HAD) group; the numbers under the columns show the number of subjects in each group as previously described. ${ }^{47}$ Notably, the CD4+ T cell counts in the HAD group (median 55, IQR 35-160 cells/ $\mu \mathrm{L}$ ) were comparable to those of the combined NA 50-199 and NA < 50 groups (median 59, intraquartile range [IQR] 20-122 cells/ $\mu \mathrm{L} ; p=0.31$, Mann-Whitney). Plasma (B) and CSF (C) HIV RNA concentrations showed different patterns of change over the subject groups, most notably in the NA group with CD4+ T cells $<50 \mu \mathrm{L}$. Elevated CSF WBC counts (D) were common in all HIV-infected groups except in the NA subjects with blood CD4+T cell counts $<50$ cells/ $\mu \mathrm{L}$ ( $p<0.01$ to $<0.0001$, Kruskal-Wallis test and Dunn'smultiple comparison test). CSF neopterin (E) was elevated in all HIV-infected groups, increased when CD4+cells fell below 350 cells $/ \mu \mathrm{L}$ and was notably elevated in the HAD group. CSF NFL (F) was high in all the HAD subjects, but also elevated in $>25 \%$ of those with CD4+ counts between 50199 cells/ $\mu \mathrm{L}$ and nearly $75 \%$ of those with CD $4+$ cells below 50 cells $/ \mu \mathrm{L}$. 


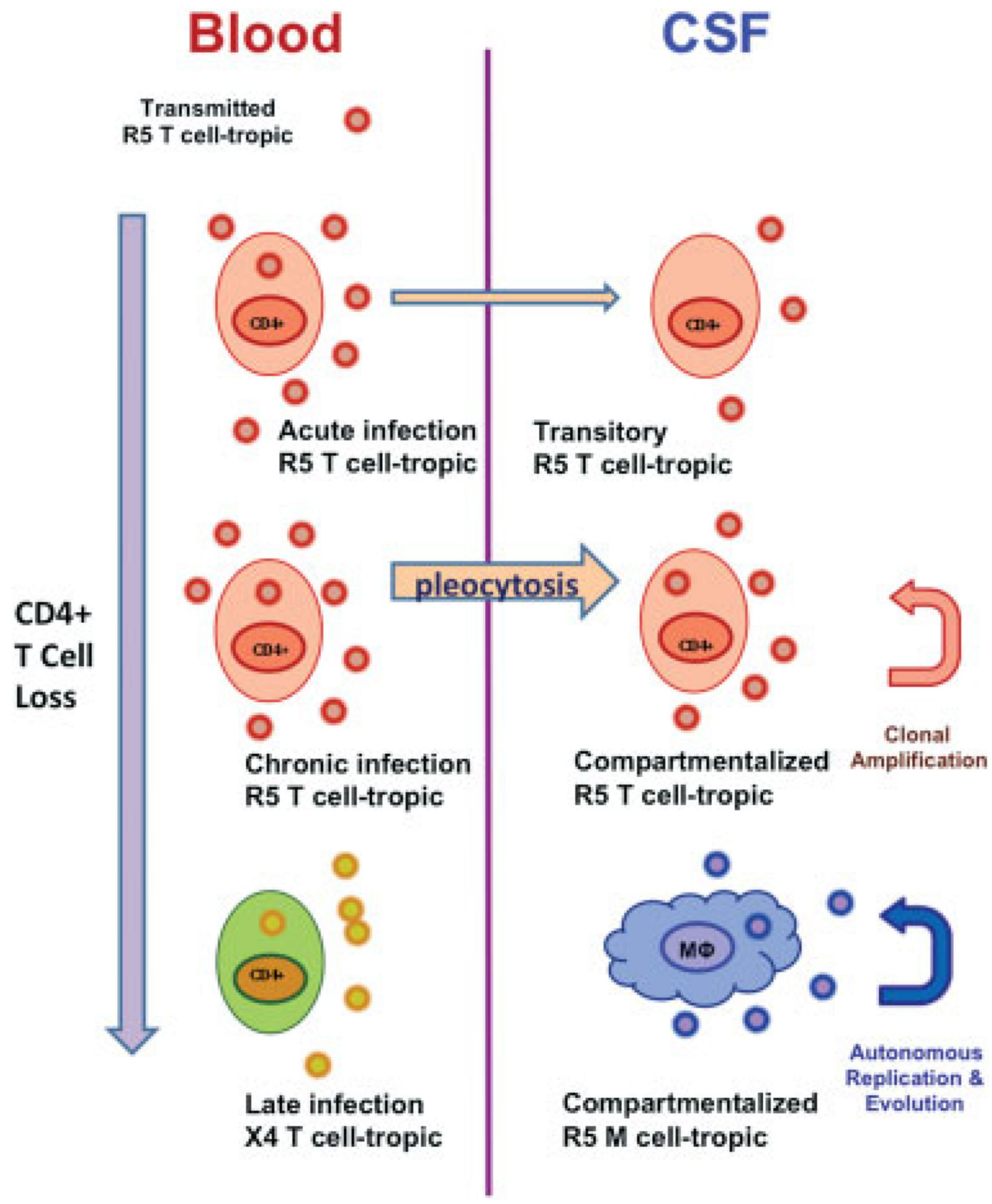

Transmitted R5 T cell-tropic

Fig. 3.

Model of types of central nervous system (CNS) infection reflected in cerebrospinal fluid (CSF). Systemic infection is shown on left and CNS on right. Acute and chronic infection by T-tropic, CCR5-using (R5) viruses generally derive from circulating systemic virus populations and likely relate to transport into the CSF by CD4+ T cells with limited or shortlived local amplification. Only occasionally do these undergo clonal amplification, one form of compartmentalization, and rarely do they further evolve and diversify locally. This contrasts to macrophage- (M-) tropic HIV-1 populations, which do not require high CD4 
levels to infect cells and can propagate within the CNS in macrophages and related cellscomprising the second and more pathogenic type of compartmentalized infection associated with HIV encephalitis and HIV-associated dementia HAD. The location and events underlying emergence of M-tropic viruses in the CNS remain uncertain. An independent evolution occurs systemically with the emergence of T-tropic CXCR4-using (X4) viruses systemically in some patients; these are associated with low-blood CD4 cells and rapid progression, and may be found in CSF. However, there is no evidence that they are directly neuropathic. In fact, R5 viruses continue to predominate in patients with low CD4 cells, but are not included in the bottom portion in this crowded figure. The figure derives from concepts developed by Swanstrom and colleagues in their work on CSF-virus phylogeny and tropism. ${ }^{30}$ 